\section{Thunderstorms, Mammato Clouds, and Globular Lightning.}

Mr. Evershed's description in NATURE of December 22 , p. 902 , of a sound emanating from a thundercloud before the storm begins is new to me: I did not hear such a sound in Simla, nor have I come across a reference to this phenomenon in the large literature on thunderstorms. I see no reason to doubt the correctness of the observation, but whether the sound has its origin in the electricity, the rain or the wind which accompanies a thunderstorm, I am unable to say, and do not wish to guess for fear of starting another long discussion in NATURE.

Mr. Dobson, in his letter on mammato clouds in NATURE of December I, p. 793, says "Any satisfactory explanation of the formation of this type of cloud would be welcome." May I accept this invitation and put forward a new explanation?

Let us imagine a column of the atmosphere with cloud above a certain height and clear air below. Now withdraw air from the foot of the column so that the whole column of air sinks. The air below the plane of separation between clear air and cloud warms up at the adiabatic rate for dry air, while above this plane the air warms up at the adiabatic rate for saturated air. These rates may be taken to be $10^{\circ} \mathrm{C}$. and $6^{\circ} \mathrm{C}$. per Iooo metres of aescent respectively. Thus the air above the plane of separation becomes relatively colder than the air below and equilibrium is disturbed, the cold cloudladen upper air tending to fall through the warm air below. If this process takes place very siowly and over a large area, the penetration of the upper cold air into the lower air will occur in the form seen in mammato clouds.

This is not the place to go into details of the process, but I may remark that the condition of the atmosphere immediately after the passage of a thunderstorm is very favourable for this process to take place. The air has recently been churned up so that the appropriate adiabatic lapse rates have been established in the cloud and in the air below, and therefore a very small downward component will quickly produce instability at the plane of separation. Also we know that there are violent ascensional currents in a thunderstorm, therefore there must be a corresponding descent of air which probably takes place as a slow settling of the whole air mass over a large area in the rear of the storm. Except in connexion with thunderstorms, appreciable downward motion within the atmosphere is extremely rare, hence mammato clouds are seldom seen except associated with thunderstorms.

While I am writing I should like to refer to one point in connexion with the discussion on globular lightning which is now taking place in NATURE. Mr. E. Kilburn Scott suggests in NATURE, November 24 , p. 760 , that " the ball may be a mass of concentrated nitrogen oxides." A similar suggestion was made in the Philosophical Magazine, volume xxi., p. 630 , by Mr. Thornton, but he suggested that the ball was composed of ozone. Now the difficulty with all such explanations is to understand how the ball is held together. If the light given off is caused by chemical combination, the temperature of the gas in the ball must be very high, and this would accelerate the natural mixing which takes place when a mass of gas is introduced into an atmosphere of another gas. I am unable to imagine any process by which a mass of hot glowing gas can hold together except in some form of vortex ring or whirl as suggested by Sir Oliver Lodge. But $I$ know of no evidence to indicate such a whirl, nor can $I$ conceive how it could be formed, while there is good evidence that the ball of globular lightning has not the nature of a flame.

Meteorological Office,

Kingsway, W.C.2,

December 24

\section{Temperature Periods in the Emission of Occluded Gases from Iron.}

IN NATURE of May I3, I922, one of us reported that, by means of thermo-electric measurements, we had found a periodicity in the properties of iron as a function of temperature such that the periods approximately coincided with the hundreds on the centigrade scale (see also Annalen der Physik, vol. 67, pp. 227 and 236 , and vol. 68, p. 67, I922). We have now discovered that the same periodicity also

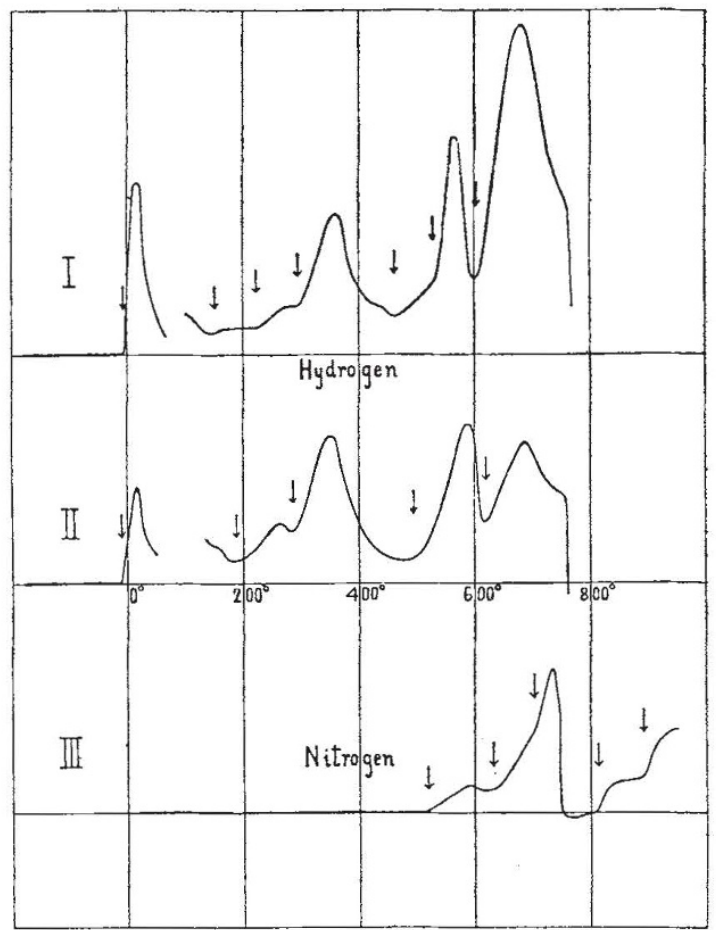

Frg. x.--Curves showing relation between pressure increase per minute and temperature for iron containing occluded hydrogen or nitrogen.

occurs when one measures the speed at which occluded gases are given off from iron upon a similar rise in temperature.

The accompanying diagram (Fig. I) shows some of the results we obtained with electrolytic iron containing occluded hydrogen or nitrogen. The specimens were placed in a quartz-tube connected to a manometer, and the apparatus was evacuated. The temperature was raised $2^{\circ}$ per minute, and the pressure was observed at intervals of two minutes. The ordinate in Fig. I is the increase of pressure per minute, taken as a mean value for twelve minutes. Curve I was obtained with a specimen having a large percentage of hydrogen. As it was chilled off with liquid air, there took place, upon the subsequent rise in temperature, an emission of gas as early as at $-5^{\circ} \mathrm{C}$.; that is, below the ordinary temperature. As the temperature rose from room temperature upwards, there occurred an accelerated emission of gas at approximately $150^{\circ}, 220^{\circ}, 300^{\circ}, 460^{\circ}, 530^{\circ}$, and $600^{\circ}$, thus with an average interval of $100^{\circ}$. The 